\title{
Morbidade psiquiátrica entre adolescentes em conflito com a lei
}

\author{
Psychiatric morbidity among adolescents in conflict with the law
}

\author{
Solange Rubim de Pinho, William Dunningham, Wania Márcia de Aguiar, Antônio de Souza \\ Andrade Filho, Katriana Guimarães, Katharine Guimarães, Taiana Rubim de Pinho Almeida \\ e Vicente de Aguiar Dunningham
}

\section{Resumo}

Objetivo: Descrever o perfil psiquiátrico, destacando os transtornos em co-morbidade entre os adolescentes em conflito com a lei da Casa de Acolhimento ao Menor (CAM), Salvador-BA, em 2003. Métodos: Estudo de corte transversal, de caráter censitário, em população de 290 jovens cumprindo medidas de privação de liberdade. Utilizaram-se questionário para identificar dados demográficos, sinais e sintomas psicopatológicos e entrevista semi-estruturada para o exame dos adolescentes. Resultados: Perfil sociodemográfico: 89,3\% sexo masculino; $63,9 \%$ entre 15 e 18 anos incompletos; $95,1 \%$ com ensino fundamental incompleto ou analfabeto; $67,6 \%$ com renda familiar menor que um salário mínimo e 54\% naturais da capital do estado da Bahia. Dos 290 indivíduos, $24,8 \%$ não apresentaram transtornos mentais e $75,2 \%$ preencheram critérios para um ou mais transtornos psiquiátricos de acordo com a décima revisão da Classificação Internacional de Doenças (CID-10). Entre os 218 jovens portadores de patologia, 47,7\% apresentaram transtornos em co-morbidade. Aassociação de patologia mais prevalente foi entre transtornos de conduta e transtornos por uso nocivo de substância psicoativa $(13,4 \%)$. Transtornos hipercinéticos só foram freqüentes quando associados a outras condições (10,7\%). 0 uso nocivo de substância psicoativa foi identificado em combinação com os diversos quadros psiquiátricos. Conclusão: Observou-se alta taxa de co-morbidade psiquiátrica, sugerindo a necessidade de estratégias terapêuticas específicas entre jovens portadores de transtornos mentais envolvidos com a justiça.

Palavras-chave: delinqüência juvenil; transtornos mentais; prevalência; co-morbidade.

\begin{abstract}
Objective: To describe the psychiatric profile, with emphasis on psychiatric comorbidities, of adolescents in conflict with the law interned on Casa de Acolhimento ao Menor (CAM), Salvador, Bahia, 2003. Methods: This is a cross-sectional census study that enrolled a population of 290 adolescents kept on a deprivation of liberty regimen. A questionnaire was used to collect demographic and psychosocial data as well as psychopathologic signs and symptoms, and a semi-structured interview was carried out on the examination of the enrolled adolescents. Results: Social-demographic profile: male (89.3\%); between 15 and 18 years $(63.9 \%)$, analphabets or had not completed the basic school years (95.1\%); monthly family income below one minimum salary (67.6\%). Among the 290 enrolled adolescents, $24.8 \%$ had no mental disturbance, while $75.2 \%$ fulfilled criteria to one or more psychiatric disorders according to ICD-10. Out of 218 adolescents who had at least one diagnosis, $47.7 \%$ had comorbidity disorders. The commonest association of conditions was between behavioral disorders and the noxious use of psychoactive drugs (13.4\%). Hyperkinetic disorders are only frequent when associated with other conditions (10.7\%). The noxious use of psychoactive drugs was also shown to be present, being noted in association with diverse psychiatric conditions. Conclusion: A high rate of psychiatric comorbidity was found, suggesting the need for specific therapeutic approaches to aid adolescents with psychiatric conditions and in conflict with the law.
\end{abstract}

Key words: juvenile delinquency; mental disorders; prevalence; comorbidity.

Fundação Bahiana para o Desenvolvimento das Ciências (FBDC) (Pinho SR) Faculdade de Medicina da Universidade Federal da Bahia (UFBA) (Pinho SR, Dunningham W, Andrade Filho AS, Aguiar WM, Guimarães K, Guimarães K, Almeida PRP, Dunningham VA)

Recebido Escola Bahiana de Medicina e Saúde Pública (EBMSP) (Andrade Filho AS)

Aprovado

Correspondência para: Wania Márcia de Aguiar

Rua Acari, 8 - Itapuã - 41610-840 - Salvador-BA - Tel: (71) 3249-0977/3359-6512 - e-mail: aguiard@uol.com.br 


\section{Introdução}

As elevadas taxas de morbidade psiquiátrica do adolescente em conflito com a lei são um fenômeno universal com singularidades demográficas, econômicas e socioculturais, e os estudos demonstram alto índice de co-morbidades nesse grupo de indivíduos.

A observação pelos pesquisadores, na sua prática cotidiana, da ocorrência de transtornos psiquiátricos entre jovens que cumprem medidas socioeducativas em instituição correcional no estado da Bahia despertou o interesse na investigação da prevalência de transtornos psiquiátricos, tipos e freqüência de co-morbidades, visando contribuir para a melhoria da assistência à saúde mental do adolescente infrator na realidade brasileira.

Na revisão bibliográfica foi limitado o número de estudos sobre o tema. Destacam-se Doreleijers et al. (2000), que encontraram alta ocorrência de transtornos diruptivos (75\%) e por déficit de atenção (58\%) entre os adolescentes cumprindo medidas de privação de liberdade em instituições correcionais. Essas mesmas patologias, somadas aos transtornos por abuso de substância psicoativa (SPA), foram as mais prevalentes nos estudos de Garland et al. (2001).

Observa-se que na adolescência é freqüente a ocorrência de dois ou mais transtornos combinados. Pesquisadores como Abram et al., (2003), Vreugdenhil et al. (2004) e Maughan et al. (2004) identificaram os transtornos isolados e apontaram para alto índice de co-morbidade em adolescentes autores de atos infracionais.

De acordo com as informações da Subsecretaria de Promoção dos Direitos da Criança e do Adolescente (2005), o Brasil contava em 2004 com 39.578 jovens infratores, 13.489 dos quais cumpriam medidas socioeducativas em regime fechado.

Levando em consideração o número crescente de jovens envolvidos com a justiça, este estudo objetivou avaliar a prevalência de transtornos psiquiátricos em adolescentes infratores, com ênfase nas co-morbidades. Para a presente investigação foi escolhida a população da Casa de Acolhimento ao Menor (CAM) de Salvador, unidade da Fundação da Criança e do Adolescente (FUNDAC) ligada à Secretaria do Trabalho e da Ação Social do Estado da Bahia.

\section{Métodos}

Realizou-se estudo censitário de corte transversal com 290 jovens infratores em regime de privação de liberdade, tomando-se como referência a décima revisão da Classificação Internacional de Doenças - Transtornos Mentais e de Comportamento (CID-10 [1998]).

Foi considerado adolescente o indivíduo que se encontrava entre os 12 e os 18 anos de idade, conforme o Estatuto da Criança e do Adolescente (ECA) (1996).

Participou da pesquisa uma equipe de cinco estagiários de psicologia e terapia ocupacional - que foram treinados nos meses de agosto e setembro de 2002 para a aplicação do questionário - e um psiquiatra. A coleta de dados iniciou-se em outubro de 2002 e teve a duração de seis meses.

Para a construção do banco de dados e a realização dos cálculos estatísticos, utilizou-se o programa Statistical Package for the Social Sciences (SPSS, 1998).
Foi utilizado o instrumento Morbidade Psiquiátrica do Adolescente em Conflito com a Lei (MPACL I e II), desenvolvido pela equipe do Grupo de Pesquisa em Saúde Mental do Centro de Estudos e Pesquisas Juliano Moreira especificamente para este estudo. O MPACL I é uma ficha para coleta de dados de identificação, queixas clínicas e tipos de delitos cometidos. Na primeira etapa da investigação, os adolescentes foram submetidos ao MPACL parte I, questionário baseado em instrumentos preexistentes (Santana, 1998) e na experiência clínica dos pesquisadores. Nessa etapa os estagiários realizaram a coleta dos dados de identificação e das queixas clínicas do adolescente. Na segunda etapa, foi aplicado o MPACL parte II, entrevista psiquiátrica semiestruturada, quando todos os indivíduos foram examinados pela autora principal, sendo atribuídos diagnósticos clínicos. O MPACL II foi elaborado com base no modelo de entrevista semi-estruturada proposto por Josef e Silva (2002). Buscou-se uma adequação do instrumento à realidade brasileira, mais precisamente a baiana.

O estudo foi aprovado pela Comissão de Ética em Pesquisa do Hospital Juliano Moreira (BA) e autorizado pelo Juizado da Infância e Adolescência de Salvador. Os adolescentes concordaram em participar assinando o termo de consentimento informado.

\section{Resultados}

A população constou de 290 jovens com idades variando entre 12 e 21 anos, média de 16,4 anos ( $\pm 1,7)$, sendo $89,3 \%$ indivíduos do sexo masculino e 10,7\% do feminino. Entre os jovens, $95,1 \%$ tinham o curso fundamental incompleto ou eram analfabetos; 0,7\%, o fundamental completo; 3,5\%, o ensino médio incompleto; 0,7\%, o completo.

A renda familiar se mostrou distribuída da seguinte maneira: $67,6 \%$ com renda familiar média inferior a um salário mínimo; 24,8\% entre um e três salários mínimos; $1,4 \%$ mais de três salários mínimos e 6,2\% não souberam informar. Quanto à procedência, $54 \%$ eram da capital do estado; $42,9 \%$, do interior; $3,1 \%$, de outras localidades.

Entre os adolescentes examinados $24,8 \%$ não preencheram critérios para transtornos mentais, enquanto $75,2 \%$ deles foram diagnosticados como portadores de transtornos psiquiátricos, incluindo os de conduta e o uso nocivo de SPA.

$\mathrm{Na}$ Tabela 1 estão expostos os dados relativos à prevalência dos transtornos psiquiátricos, isolados e em co-morbidade. Dentro do universo populacional de 290 jovens, observa-se que os transtornos em co-morbidade ocorreram em uma taxa considerada elevada quando comparada à dos adolescentes com transtorno psiquiátrico isolado.

Tabela 1. Prevalência de transtornos psiquiátricos, isolados e em co-morbidade, e ausência de doença em adolescentes no CAM em 2003

\begin{tabular}{lcc}
\hline Transtorno & $\boldsymbol{n}$ & $\%$ \\
\hline Isolado & 114 & 39,31 \\
Em co-morbidade & 104 & 35,86 \\
Sem transtorno & 72 & 24,83 \\
\hline
\end{tabular}

CAM: Casa de Acolhimento ao Menor. 
$\mathrm{Na}$ Tabela 2, que contém as patologias isoladas, destacam-se os transtornos de conduta seguidos do retardo mental, os por uso nocivo de SPA e os outros transtornos psicóticos como as patologias que apresentaram as maiores taxas de prevalência de transtornos isolados.

Tabela 2. Distribuição da prevalência dos transtornos psiquiátricos isolados por categoria diagnóstica no CAM em 2003

\begin{tabular}{lcc}
\hline Categoria & $\boldsymbol{n}$ & $\%$ \\
\hline T. de conduta & 43 & 14,8 \\
T. hipercinéticos & 2 & 0,7 \\
T. por uso nocivo de SPA & 15 & 5,2 \\
Outros t. psicóticos & 14 & 4,8 \\
Esquizofrenia & 5 & 1,7 \\
Retardo mental & 20 & 6,9 \\
Estados ansiosos & 7 & 2,4 \\
Estados depressivos & 3 & 1 \\
T. mentais orgânicos & 5 & 1,7 \\
\hline
\end{tabular}

CAM: Casa de Acolhimento ao Menor; T: transtorno(s); SPA: substância psicoativa.

A Figura 1 mostra a distribuição da morbidade psiquiátrica da população estudada por categorias nosológicas isoladas e com as suas co-morbidades. Chamam a atenção a prevalência do transtorno de conduta, o transtorno por uso nocivo de SPA, o retardo mental e os transtornos hipercinéticos.

Figura 1. Prevalência dos transtornos psiquiátricos isolados e em co-morbidade, por categoria diagnóstica, na população geral (290 indivíduos) do CAM em 2003

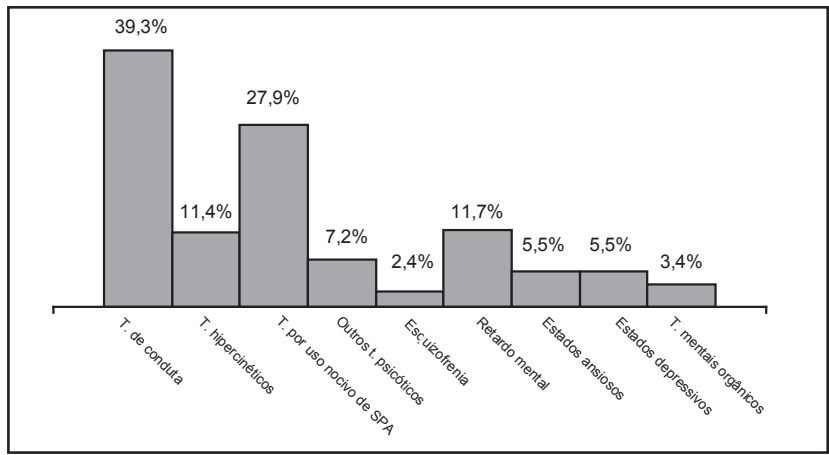

CAM: Casa de Acolhimento ao Menor; T: transtorno(s); SPA: substância psicoativa.

Considerando-se o total de adolescentes portadores de transtornos psiquiátricos (218 indivíduos), observou-se que $52,35 \%$ apresentaram transtornos psiquiátricos isolados e $47,7 \%$, transtorno em co-morbidade.

Analisando-se as patologias mais prevalentes e os transtornos co-mórbidos na população de 290 indivíduos, verifica-se maior ocorrência dos transtornos de conduta associados aos por uso nocivo de SPA e transtornos de conduta associados aos transtornos hipercinéticos (Tabela 3 ).
Tabela 3. Prevalência dos transtornos de conduta isolados e em co-morbidade no CAM em 2003

\begin{tabular}{lcc}
\hline Patologia & $\boldsymbol{n}$ & $\%$ \\
\hline T. de conduta isolado & 43 & 15 \\
T. de conduta + t. por uso nocivo de SPA & 39 & 13,5 \\
T. de conduta + t. hipercinéticos & 18 & 6,2 \\
T. de conduta + t. hipercinéticos + t. por uso & 9 & 3,1 \\
nocivo de SPA & & \\
\hline
\end{tabular}

CAM: Casa de Acolhimento ao Menor; T: transtorno(s); SPA: substância psicoativa.

Os transtornos por uso nocivo de SPA são os que aparecem mais uniformemente combinados com os demais (Tabela 4), sendo o mais importante transtorno co-mórbido.

A entidade clínica retardo mental não teve índices representativos de co-morbidades. Foi, isoladamente, responsável por uma prevalência já elevada de 7,3\%, destacando-se apenas a associação com outros transtornos psicóticos $(1,7 \%)$ e com aqueles por SPA $(1,4 \%)$.

$\mathrm{O}$ transtorno hipercinético, quando considerado isoladamente, apresentou baixa prevalência $(0,7 \%)$, contudo, quando associado a outras patologias, representou 10,7\%, taxa que merece ser destacada (Tabela 5).

Tabela 4. Prevalência dos transtornos por uso nocivo de SPA isolados e em co-morbidade no CAM em 2003

\begin{tabular}{lcc}
\hline Patologia & $\boldsymbol{n}$ & $\%$ \\
\hline T. por uso nocivo de SPA + t. de conduta & 39 & 13,5 \\
T. por uso nocivo de SPA isolado & 15 & 5,2 \\
$\begin{array}{l}\text { T. por uso nocivo de SPA + t. de conduta + t. } \\
\text { hipercinéticos }\end{array}$ & 9 & 3,1 \\
$\begin{array}{l}\text { T. por uso nocivo de SPA + estados depres- } \\
\text { sivos }\end{array}$ & 6 & 2,1 \\
T. por uso nocivo de SPA + retardo mental & 5 & 1,7 \\
\hline
\end{tabular}

CAM: Casa de Acolhimento ao Menor; T: transtorno(s); SPA: substância psicoativa.

Tabela 5. Prevalência dos transtornos hipercinéticos isolados e em co-morbidade no CAM em 2003

\begin{tabular}{lll}
\hline Patologia & $\boldsymbol{n}$ & $\%$ \\
\hline T. hipercinéticos + t. de conduta & 18 & 6,3 \\
T. hipercinéticos + t. de conduta + t. por uso & 9 & 3,1 \\
nocivo de SPA & 2 & 0,7 \\
T. hipercinéticos + t. por uso nocivo de SPA & 2 & 0,7 \\
T. hipercinéticos isolados &
\end{tabular}

CAM: Casa de Acolhimento ao Menor, T: transtorno(s); SPA: substância psicoativa.

\section{Discussão}

A presente investigação traz resultados importantes no que diz respeito à prevalência de transtornos psiquiátricos entre os jovens em conflito com a lei da CAM, incluindo os transtornos em co-morbidade. Dos 290 indivíduos pesquisados, $75,2 \%$ preencheram critérios diagnósticos para transtornos psiquiátricos, isolados ou em co-morbidade, demonstrando a associação entre comportamento infrator e transtorno psiquiátrico na adolescência. Quando considerada a população de jovens com transtornos psiquiátricos $(n=218)$, 
identificaram-se $47,7 \%$ com diagnósticos duplos ou triplos. Esse dado corrobora os da literatura internacional, em que os indivíduos dessa faixa etária aparecem como portadores de transtornos combinados.

Abram et al. (2003), na pesquisa realizada no Centro de Detenção Juvenil Cook County Illinois, encontraram na amostra investigada $56,5 \%$ de indivíduos do sexo feminino e $45,9 \%$ do masculino, preenchendo critérios para dois ou mais dos seguintes transtornos: depressão maior, distimia, mania, psicoses, transtorno de conduta, transtorno de oposição desafiante, transtornos de ansiedade, transtornos por déficit de atenção e comportamento diruptivo e transtornos por uso de substância.

Apoiando os resultados da presente investigação, Maughan et al. (2004), ao pesquisarem um universo de 18.760 jovens criminosos (incluindo indivíduos em liberdade provisória e sentenciados considerados culpados) segundo registros oficiais do Reino Unido, afirmaram que, na prática, a maioria apresentou múltiplos distúrbios mentais. Detectaram a existência de um ou mais distúrbios psiquiátricos em $95 \%$ dos jovens por eles pesquisados.

Ainda em relação à co-morbidade, Doreleijers et al. (2000) estudaram 108 adolescentes em privação de liberdade: $75 \%$ apresentaram no mínimo um diagnóstico; $46 \%$, dois e $17 \%$, três diagnósticos.

$\mathrm{Na}$ pesquisa da CAM as maiores prevalências de transtornos em co-morbidades foram: transtorno de conduta associado ao por uso nocivo de SPA e transtorno de conduta combinado com transtorno hipercinético, assim como nos estudos de Garland et al. (2001), Abram et al. (2003) e Maughan et al. (2004). Esses autores encontraram índices mais altos do que os identificados na CAM, o que pode ser atribuído a fatores regionais e de ordem metodológica.

Outros estudos apontam a coexistência do comportamento anti-social e dos transtornos psiquiátricos na infância e na adolescência. Em 2000, o National Institute of Mental Health (NIMH), em artigo de revisão, também abordou a associação entre delinqüência, transtornos de conduta e transtornos hipercinéticos, igualmente identificada na CAM.

Em relação ao transtorno de conduta, freqüentemente os jovens com esse diagnóstico não são considerados portadores de patologia psiquiátrica, mas pessoas que não internalizaram normas e desafiam os limites socialmente determinados. Os jovens com transtorno de conduta manifestam-se com sentimento deficitário de empatia pelo próximo e ausência de culpa, que atuam como facilitadores para a incursão no crime.

Os transtornos por uso nocivo de SPA na CAM se associaram às mais diversas patologias (22,4\%): transtornos de conduta, transtorno hipercinético, esquizofrenia, outras psicoses, estados ansiosos e estados depressivos. Isso permite inferir que o uso de SPA desempenha papel importante na determinação do comportamento infrator por levar adolescentes a perderem a capacidade de controlar seus impulsos, enquanto alguns entram no comércio ilegal, passando a con- viver com ameaças de morte, fuga de casa, furtos, assaltos, tráfico de drogas e outros delitos. Quanto ao retardo mental, acredita-se que, devido à dificuldade de adaptação concreta à realidade dos jovens portadores desses transtornos, eles não são freqüentemente atores principais de atos infracionais. Entretanto a ocorrência de $7,3 \%$ de portadores de retardo mental nessa instituição sugere que eles participam de delitos praticados por outros indivíduos e em uso de substâncias psicoativas (Abdalla-Filho, 2004).

Os transtornos hipercinéticos, cujo sintoma de impulsividade é um dos mais importantes em co-morbidade (10,7\%), fazem prever maior vulnerabilidade ao comportamento agressivo, especialmente nas associações com os transtornos de conduta e por uso nocivo de SPA. As co-morbidades para esses transtornos de maior relevância no presente trabalho foram com o transtorno de conduta $(6,3 \%)$ e a tríplice associação com transtornos de conduta e por uso nocivo de SPA $(3,1 \%)$. Os resultados do estudo de Andrade e Assumpção Júnior (2004), em Governador Valadares, revelaram número muito maior de casos de transtorno de déficit de atenção (equivalente na presente investigação, pela CID-10 [1998], aos transtornos hipercinéticos) em menores envolvidos com a justiça do que os detectados na CAM. Tal fato pode ser atribuído às características de cada população; às metodologias aplicadas; à dificuldade para o diagnóstico diferencial com transtornos de conduta e de aprendizagem, retardo mental leve, estados depressivos e ansiosos, além da existência de diagnósticos duplos e triplos. No caso específico da pesquisa, na Bahia, a essas dificuldades somou-se a impossibilidade de informações detalhadas sobre o desenvolvimento do indivíduo, bem como a falta de contato com a família e a ausência de dados sobre o desempenho escolar do adolescente.

\section{Conclusão}

$\mathrm{Na}$ presente investigação foi verificada associação entre comportamento infrator e transtorno psiquiátrico. Entre os transtornos, chama a atenção a freqüência de jovens com os transtornos de conduta, patologia na qual o fator social tem peso relevante. Entre os 218 jovens portadores de patologia, $47,7 \%$ apresentaram transtornos em co-morbidade e $52,3 \%$, transtornos isolados. Transtornos de conduta, por abuso de SPA, retardo mental e hipercinéticos, em co-morbidade, foram os mais prevalentes nesta investigação. Tais dados sugerem a importância de procedimentos diagnósticos mais apurados na adolescência, utilizando instrumentos para a triagem e definindo precocemente o encaminhamento terapêutico mais adequado para cada indivíduo.

0 adolescente portador de enfermidade psiquiátrica, cuja patologia tem nexo causal com o delito, necessita de tratamento médico, psicológico, bem como de um trabalho orientado para a sua reintegração social. É fundamental ainda 0 acesso facilitado aos bens e aos serviços coletivos. Sabese que confinar, por si só, não evita nem trata, devendo as intervenções ser múltiplas e combinadas. 


\section{Referências}

Abdalla-Filho E. Retardo mental. In: Taborda, JGV, Chalub M, Abdalla-Filho E, organizadores. Psiquiatria Forense. Porto Alegre: ArtMed; 2004.

Abram KM, Teplin LA, McClelland GM, Dulcan MK. Comorbid psychiatric disorders in youth in juvenile detention. Arch Gen Psychiatry, 60(11): 1097-108, 2003

Andrade RC, Silva VA, Assumpção Júnior FB. Preliminary data on the prevalence of psychiatric disorders in Brazilian male and female juvenile. Braz J Med Biol Res, 37(8): 1155-60, 2004.

Doreleijers TAH, Moser F, Thijs P, Engeland HV, Beyaert FHL. Forensic assessment of juvenile delinquents: prevalence of psychopathology and decision making at court in the Netherlands. J Adolesc, 23: 263-75, 2000

Garland AE, Hough RL, McCabe KM, Yeh M, Wood PA, Aarons GA Prevalence of psychiatric disorders in youths across five sectors of care [editorial]. J Am Acad Child Adolesc Psychiatry, 40(4): 409-18, 2001.

Josef F, Silva A. Psiquiatria forense no Brasil: modelo de entrevista semiestruturada para emprego em pesquisa psiquiátrico-forense, com utilização do Hare PCL-R. J Bras Psiquiatr, 51(1): 47-54, 2002.

Maughan B, Brock A, Ladva G. Mental health. In: National Mental Health Association. The health of children and young people. Reino Unido:
Office for National Statistics, 2004. Disponivel em:<http:// www.nso. gov.nt/pdf>.

National Institute of Mental Health. Child and Adolescent Violence research at the NIMH 2000. Disponivel em: <http:/www.nimh>.

Norusis MJ. Statistical Package for the Social Sciences (SPSS). Chicago 1998.

Oliveira J, organizador. Estatuto da Criança e do Adolescente. 7 ed. São Paulo: Saraiva; 1996.

Organização Mundial da Saúde. Classificação de transtornos mentais e de comportamento da CID-10: critérios diagnósticos para pesquisa. Porto Alegre: Artes Médicas; 1998.

Rede Internacional Solitária. Situação do sistema socioeducativo - 2004 Disponivel em: <http://www.risolidaria.org.br>. Acesso em: 15 set 2005.

Santana VS. Estudo epidemiológico das doenças mentais em um bairro de Salvador, Nordeste de Amaralina [dissertação]. Salvador: Universidade Federal da Bahia; 1978.

Vreugdenhil C, Doreleijers TA, Verneire R, Wouters LF, van den Brink $W$. Psychiatric disorders in a representative sample of incarcerated boys in the Netherlands. J Am Acad Child Adolesc Psychiatriy, 43(1): $97-104,2004$ 